Bangladesh J. Zool. 40(2): 213-220, 2012

\title{
DIAGNOSIS OF TUBERCULOSIS AND RISK FACTORS INVOLVED
}

\author{
Hamida Khanum*, Rumana Sultana and Ripon Chandra Dhar \\ Department of Zoology, University of Dhaka, Dhaka-1000, Bangladesh
}

\begin{abstract}
The present study was carried out over a total of 330 suspected outdoor TB patients from the Shyamoli Chest Clinic, Dhaka. The prevalence of TB was $29.69 \%$, male and female ratio for the prevalence being 2.4:1. The highest prevalence (18.37\%) was observed among the 20-24 age group followed by 25-29 age group (17.35\%). Prevalence of pulmonary tuberculosis (PTB) among the total cases was $74.51 \%$; on the other hand, extra-pulmonary tuberculosis (EPTB) was $25.51 \%$. According to the severity of 73 PTB cases, $52.05 \%$ had severe pulmonary infection, $28.8 \%$ moderate pulmonary infection, and $19.2 \%$ mild TB infection. Among 98 TB positive cases, $14.29 \%$ had relapse cases and $85.71 \%$ had new cases. Among the 14 relapse cases, $85.71 \%$ patients had PTB while $14.29 \%$ patients had EPTB. On the other hand, out of 84 new cases, $72.62 \%$ had PTB and $27.38 \%$ had EPTB. According to occupation, the highest prevalence (21.43\%) was observed among service holders, followed by garments workers and laborers $(17.35 \%)$. The lowest prevalence $(9.18 \%)$ was found among unemployed people. The higher prevalence of TB was mostly associated with poor socio-economic condition, close contact with infected cases, illiteracy, gender related factors and health care.
\end{abstract}

Key words: Prevalence, tuberculosis, sputum, pulmonary tuberculosis, extrapulmonary tuberculosis

\section{INTRODUCTION}

Tuberculosis (TB) is a contagious air borne infection caused by the bacterium Mycobacterium spp. (mostly Mycobacterium tuberculosis and occasionally $M$. bovis) which is passed when an infected person coughs, sneezes, talks, or spits and another person inhales the infected air. TB most often affects the lungs, but it can also affect the viscera like lymph nodes, bones, joints, skin, the central nervous system, the urinary tract and the abdomen, or it can give a disseminated form of disease (miliary tuberculosis). Individuals infected with TB can have latent TB infection or active TB disease. Those with latent TB infection do not exhibit symptoms and cannot spread the infection to others, whereas those with active TB disease exhibit a range of symptoms and are contagious. $M$. tuberculosis is a rod shaped, non-motile slow-growing facultative intracellular parasite, able to multiply inside the macrophage phagosome, in which the environment is generally hostile for most bacteria and can also replicate extracellularly in the open lung cavities (Manganelli et al. 1999).

*Author for correspondence. E-mail: hamida_khanum@yahoo.com 
Tuberculosis remains a major worldwide health concern and has been characterized as one of the three epidemics by the World Health Organization (Dou et al. 2011). Over the past two decades, the number of tuberculosis cases has been risen worldwide, especially in the developing countries of Southeast Asia and sub-Saharan Africa, where co-infection with human immunodeficiency virus (HIV) and tuberculosis is common (Kendall 2012). With a population of 150 million, Bangladesh ranks sixth among the countries with a high TB burden and ranks $5^{\text {th }}$ among 22 countries with global burden of TB (WHO 2007).

In Bangladesh, TB control program is implemented in partnership with NGOs. Several private and corporate sectors are involved in TB control and in rendering services in line with international standards for TB care. Thirty seven public hospitals including medical college hospitals and military hospitals have been involved so far and services have also been established in the prison system (WHO 2009).

The laboratory diagnosis and other investigations are pre-requisite to clinical management, epidemiological investigation required for treatment as well as prevention of TB. So, the present study was intended to diagnose TB in two distinct phases.

\section{MATERIAL AND METHODS}

The study was carried out at Shamoli Chest Disease Clinic, Dhaka from January to December 2006. A total of 330 cases from all ages of both sexes was included in the study. The specimens were collected from the outdoor patients and examined in the laboratory. Related clinical information was obtained through a brief interview of the patients.

Suspected patients were asked to give three sputum specimens which were collected within three consecutive days. If it was not possible, the first specimen was taken at the first interview; the second was collected next morning at patient's home and the third on the next working day. Hospitalized patients could deliver early morning specimen on three consecutive days.

Sputum collection took place in a well ventilated room, but never in the laboratory. Samples were collected in clean, sterile, leak-proof, wide-mouth containers. The patients were requested to rinse their mouth with fresh and clean water to wash any food particles remaining in their mouth. Then, they were demonstrated how to give a good sputum sample. They were asked to cough deeply followed by repeated deep breath. To avoid contamination and infection no one was allowed standing in front of the patient. After collection, the sputum container was labeled appropriately. 
Tuberculosis was diagnosed in two distinct phases as follow: 1) Diagnosis of pulmonary tuberculosis (PTB) - All samples were smeared and stained using Ziehl-Neelsen method and stained smears were examined with $100 \mathrm{X}$ oil immersion lenses. The number of Acid-Fast Bacilli (AFB) seen in the smear was indicative of disease severity and patient's infectivity; 2) Diagnosis of extrapulmonary tuberculosis (EPTB) - If the sputum examinations were negative, but the symptoms of TB already exist, the patients were treated as EPTB patients because EPTB occurs in visceral organs other than the lungs and are not detected in sputum smears. Then the EPTB patients were subjected to other diagnostic approaches like chest X-ray, tissue biopsy, barium examination etc.

\section{RESULTS AND OBSERVATION}

Out of 330 patients, 98 (29.69\%) were found positive for Mycobacterium spp. and $212(64.24 \%)$ were male and $118(35.26 \%)$ were female. Among the males 69 $(70.41 \%)$ were infected while, $29(29.60 \%)$ females were found infected. The ratio of the prevalence of TB between male and female was 2.4:1 (Table 1).

Table 1. Prevalence of TB according to sex of the patients attended in a chest clinic in Dhaka between January and December 2006.

\begin{tabular}{lccc}
\hline Sex & Total patient & Number of positive cases & Prevalence (\%) \\
\hline Male & 212 & 69 & 32.54 \\
Female & 118 & 29 & 24.57 \\
\hline
\end{tabular}

The highest prevalence (18.37\%) was observed in $20-24$ age group followed by $17.35 \%$ in age group of $25-29$. The lowest prevalence $(3.06 \%)$ was observed in the 55-59 age group patients (Table 2).

Table 2. Prevalence of TB according to age of the patients attended in a chest clinic in Dhaka between January and December 2006.

\begin{tabular}{cccc}
\hline Age group (Years) & Total patient & Number of positive cases & Prevalence (\%) \\
\hline $10-14$ & 20 & 4 & 4.08 \\
$15-19$ & 36 & 13 & 13.27 \\
$20-24$ & 44 & 18 & 18.37 \\
$25-29$ & 63 & 17 & 17.35 \\
$30-34$ & 26 & 9 & 9.18 \\
$35-39$ & 19 & 7 & 7.14 \\
$40-44$ & 36 & 7 & 7.14 \\
$45-49$ & 16 & 5 & 5.10 \\
$50-54$ & 21 & 5 & 5.10 \\
$55-59$ & 8 & 3 & 3.06 \\
$60+$ & 41 & 10 & 10.20
\end{tabular}

Out of 98 positive cases, $73(74.51 \%)$ were smear positive and hence were treated as PTB, rest $25(25.51 \%)$ being EPTB. Out of 212 male patients, 54 
(25.47\%) were PTB patients and $19(16.10 \%)$ female patients were PTB positive; whereas, $15(7.08 \%)$ male and $10(8.47 \%)$ females were EPTB patients (Table 3). Thus, PTB was increasing alarmingly from mild to severe irrespective of sex.

Table 3. Distribution of PTB and EPTB among the male and female patients attended in a chest clinic in Dhaka between January and December 2006.

\begin{tabular}{lccccc}
\hline Sex & $\begin{array}{c}\text { Total } \\
\text { patient }\end{array}$ & $\begin{array}{c}\text { Number of PTB } \\
\text { positive cases }\end{array}$ & $\begin{array}{c}\text { Prevalence } \\
(\%)\end{array}$ & $\begin{array}{c}\text { Number of EPTB } \\
\text { positive cases }\end{array}$ & $\begin{array}{c}\text { Prevalence } \\
(\%)\end{array}$ \\
\hline Male & 212 & 54 & 25.47 & 15 & 7.08 \\
Female & 118 & 19 & 16.10 & 10 & 8.47 \\
\hline
\end{tabular}

Among 73 PTB patients, 38 (52.05\%) had severe, 21 (28.8\%) had moderate and $14(19.2 \%)$ had mild pulmonary infections (Table 4).

Table 4. Prevalence of PTB according to severity of cases.

\begin{tabular}{lcc}
\hline Type of PTB & Number of cases & Prevalence $(\%)$ \\
\hline Severe & 38 & 52 \\
Moderate & 21 & 28.8 \\
Mild & 14 & 19.2 \\
\hline
\end{tabular}

Among 25 EPTB patients, the distribution of infection was found in various locations as demonstrated in Fig. 1. Cervical lymph node TB $(28 \%)$, Intestinal TB (12\%), Pleural TB (12\%), Gland TB (20\%), Spinal TB (12\%) and Joint TB (16\%) were observed. Cervical lymph node TB was found highly prevalent followed by Gland TB (Fig. 1).

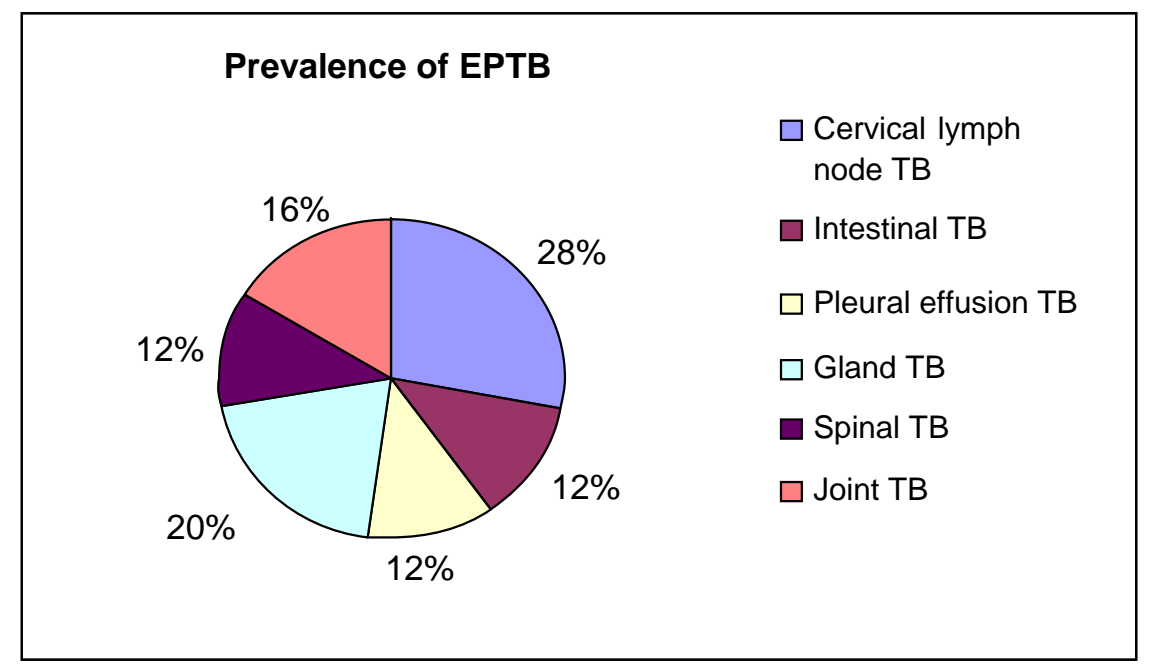

Fig. 1. Distribution of EPTB according to location. 


\section{DISCUSSION}

The present study revealed that PTB was more prevalent than EPTB. The most frequent form of presentation of TB is a disease that affects the lungs (PTB), while less frequent forms may affect any part of the body (EPTB) or present as acute disseminated TB. PTB is the major cause of morbidity and mortality which is prevalent in the developing world. Individual living in contact with smear-positive cases have a risk that is directly proportional to their contact with the patient. The greatest risk is observed among the individuals who live in the same household as a smear-positive PTB cases. In PTB, the extent of the disease can vary from minimal to massive involvement, but without effective therapy, the disease becomes progressive.

The consequences of TB in society are immense. World-wide, one person out of three, is infected with TB holding the $7^{\text {th }}$ place in the global ranking of cause of death and unless intensive efforts are made, it is likely to maintain that position through to 2020, despite a substantial projected decline in disease burden from other infectious diseases (Dye 1999, Smith et al. 2004).

Tuberculosis is one of the major public health problems in Bangladesh. So, the proper diagnosis of the disease status, prevention and control of TB is very essential. As such, with a view to help adopting a suitable strategy to combat the situation by National Tuberculosis Control Program, the study has been conducted to determine the prevalence of TB in the study area.

Effective drugs to treat and cure the disease have been available more than 50 years, yet every 10 seconds, someone in the world dies from TB, even more alarming that a person is newly infected with $M$. tuberculosis every second of every day; and left untreated, a person with active TB will infect an average of 10-15 other people every year (Dye et al. 2005). TB affects people of all age groups; children as well as adults are victim of the disease. It hinders the socioeconomic and familial development. In the $95 \%$ of all cases, $99 \%$ of death occur in developing countries, with the greatest burden in sub-Saharan Africa and Southeast Asia. Household costs of TB are substantial (Dye 2006). Furthermore, the age group, which corresponds to a period of sexual activity, is mosty exposed to the risk of HIV infection, which explains the increase in the number of TB cases in certain high TB prevalence countries heavily affected by HIV epidemic.

Under the National Tuberculosis Control Program, Bangladesh, the diagnosis of TB has been categorized into four quarters in a year; the highest prevalence (91.4\%) was observed in the $3^{\text {rd }}$ quarter (July-September) and lowest $(85.57 \%)$ in the $1^{\text {st }}$ quarter (January-March). In Kuwait, the un-adjusted observed monthspecific proportion of TB cases (per 100,000) among migrants was highest in March and lowest in October (Akhter et al. 2008). Twelve studies conducted 
between the period 1971 and 2006 from 11 countries/regions around the world (namely, South Western Cameroon, South Africa, India, Hong Kong, Japan, Kuwait, Spain, UK, Ireland, Russia, and Mongolia) were reviewed and the seasonal pattern of tuberculosis with a mostly predominant peak was seen during the spring and summer seasons in all of the countries (except South Western Cameroon and Russia) (Fares 2011).

In most countries, tuberculosis (TB) notification is twice as high in men as in women. Sex-specific features of nutrition and metabolism may also be associated with susceptibility or resistance to $M$. tuberculosis. In the study $74.51 \%$ were found PTB and rest $25.51 \%$ were EPTB in which $25.47 \%$ male patients were PTB patients and $16.10 \%$ female patients were PTB positive; whereas $7.08 \%$ male and $8.47 \%$ female were EPTB patients. Men seem to be more affected than women, with a male/female ratio of 1.9:1.0 for the worldwide case notification rate. In some countries this ratio may reach values as high as 3:1 in Armenia, for instance, but ratios below one are extremely rare and mostly correspond to very small populations of patients. This excess of male pulmonary TB cases is seen in all regions of the world, and in almost all countries, at least in non-HIV-infected patients (Neyrolles et al. 2009).

In this study, among PTB patients $52.05 \%$ had severe, $28.8 \%$ had moderate and $19.2 \%$ had mild pulmonary infection. The severity of the disease might vary in different areas within the country and in different age groups irrespective of sex.

Among EPTB patients, the distribution of infection was found in various locations. Cervical lymph node TB (28\%), Intestinal TB (12\%), Pleural TB (12\%), Gland TB (20\%), Spinal TB (12\%) and Joint TB (16\%) were observed. Cervical lymph node TB was found highly prevalent followed by Gland TB. Earlier in Bangladesh extra-pulmonary tuberculosis was found Lymph node tuberculosis was $36.2 \%$, abdominal tuberculosis $35 \%$. Cervical lymph nodes alone $(37.9 \%)$ were commonly affected among the lymph node tuberculosis (Karim et al. 2006). There is no single factor responsible for the emergence of this disease, but rather the interaction of multifactor. A multitude of risk factor for the progression from sub-clinical latent infection with $M$. tuberculosis to overt disease has been identified; the three most important for all practical purposes are co-infection with HIV, recent infection tubercle bacilli, and healed lessons from previous TB which was never treated. But, HIV infection, an important risk factor for TB worldwide, has not been widespread in Bangladesh.

Malnourished population seems to have greater likelihood of developing disease. There is evidence that individual remain infected even after spontaneous resolution of symptoms and if they become immune compromised. 
They may develop TB infection. In Germany, TB mortality increased rapidly during the First World War, declined subsequently and increased again during the period of monetary inflation with serious food shortage in the year 19221923.

Smoking may be one of the risk factor for TB prevalence. Two studies in England expanded the relationship between cigarette smoking and the risk of TB (Edwards 1957). From this case control studies there is evidence that the odds of TB increase with an increase number of cigarette smoked. In a study in Shanghai, TB incidence was shown to be higher among smoker than nonsmoker.

Finally it can be concluded that, TB is creating an extra load on the major public health of Bangladesh which is expanding due to over population. This leads to a serious economic loss to the country. This disease hampering the productivity and vitally needed socioeconomic progress, as TB kills more than any infectious disease; if left untreated, a person with active TB will infect an average of 10-15 other people every year (Dye et al. 2005).

In Bangladesh, direct sputum smear microscopy remains the most cost effective tool for the diagnosis of TB and for the monitoring progress of treatment. Clinical signs and symptoms, socio-economic conditions of the patients, their previous case diagnosis and transmission history was also observed.

TB remains a major cause of morbidity and mortality in Bangladesh (Zaman et al. 2007). According to the health ministry statistics, one person dies of TB every $10 \mathrm{~min}$ and one is infected every two minutes in the country (Mahmood 2010). This severe situation has placed Bangladesh in the sixth position in the world in terms of burden of TB patients (Mahmood 2010 and Zaman et al. 2007). So National TB Control Programme (NTP) needs more community participation and further expansion of its activities. The NTP continued its activities under a reinstated separate directorate for mycobacterial disease control (WHO 2007).

\section{LITERATURE CITED}

AKHTAR, S. and MOHAMMAD, H. G. H. H. 2008. Seasonality in pulmonary tuberculosis among migrant workers entering Kuwait. BMC Infectious Diseases 8: 3.

CDCP. 2011. Centers for Disease Control and Prevention. Health Information for International Travel 2011, The Yellow Book. USA.

DOU, H.Y., HUANG, S.C. and SU, I.J. 2011. Prevalence of Mycobacterium tuberculosis in Taiwan: Model for Strain Evolution Linked to Population Migration. International Evol. Biol. 6: 
DYE, C. 1999. Global Burden of tuberculosis: estimated incidence, prevalence and mortality by country. J. Am. Me. Assoc. 282: 677-686.

DYE, C., WATT, C. J., BLEED, D. M., HOSSEINI, S. M. and RAVIGLIONE, M. C. 2005. Evolution of Tuberculosis Control and Prospects for Reducing Tuberculosis Incidence, Prevalence, and Deaths Globally. JAMA 293(22): 2767-2775.

DYE, C. 2006. Global epidemiology of tuberculosis. Lancet 367: 938-940.

EDWARDS, J.H. 1957. Contribution of cigarette smoking to respiratory disease. Br. J. Prev. Soc. Med. 11: 10-21.

FARES, A. 2011. Seasonality of Tuberculosis. J. Glob. Infect. Dis. 3(1): 46-55.

KARIM, M. M., ChOUdhURY, S. A., HUSAIN, M. M. and FAIZ, M. A. 2006. A Clinical Study on Extrapulmonary Tuberculosis. J. Bangladesh Coll. Phys. Surg. 24 (1): 19-28.

KENDALL, A. E. 2012. U.S. Response to the Global Threat of Tuberculosis: Basic Facts. CRS Report for Congress, Congressional Research Service.

MAHMOOD, S. A. I. 2010. TB and HIV/AIDS in Bangladesh. J. AIDS HIV Res. 2(4): 66-78.

MANGANELLI, R., DUBNAU, E., TYAGI, S., KRAMER, F.R. and ITH, I. 1999. Differential expression of 10 sigma factor genes in Mycobacterium tuberculosis. Mol. Microbiol. 31: 715-724.

NEYROLLES, O. and QUINTANA-MURCI, L. 2009. Sexual Inequality in Tuberculosis. PLoS Med. 6(12):

SMITH, C. V., SHARMA, V. and SACCHETTINI, J. C. 2004. TB drug discovery: addressing issues of persistence and resistance. Tuberculosis 84(1): 45-55.

USAID Bangladesh 2009. [On- line]. Bangladesh. Available: http://www.usaid.gov/our_work/ global_health/id/tuberculosis/countries/asia/bangladesh.pdf. Accessed: Feb 7, 2010.

WHO. 2007. National Tuberculosis Control Programme, Bangladesh. Report of the Fourth Joint Review. WHO Project: BAN TUB 6: 66.

WHO. 2009. TB in South-East Asia. WHO Regional Office for South-East Asia.

ZAMAN, K., HOSSAin, S., YUNUS, M., ARIFEen, S. E., MAHMUd, A., BEGUM, V., ISLAM, A., BAQUI, A. H. and LUBY, S. 2007. Tuberculosis in Bangladesh: A 40-Year Review .11 ASCON. ICDDR,B. Scientific session, abstract book 86: 4-6 March 2007. 